\title{
Use of open stent grafting for a mycotic aortic arch aneurysm
}

Hideyuki Iwaki, MD, ${ }^{a}$ Seko Suzuki, MD, ${ }^{a}$ Toshimi Yajima, MD, ${ }^{b}$ Shigeo Yamauchi, MD, ${ }^{\text {b }}$ Masami Ochi, MD, ${ }^{\text {b }}$ and Kazuo Shimizu, MD, Okinawa and Tokyo, Japan

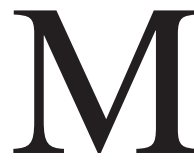

ycotic aneurysms of the thoracic aorta are uncommon. We experienced a patient with a mycotic aneurysm of the distal aortic arch, and good results were obtained with open stent grafting without a resection of the aneurysm.

\section{Clinical Summary}

A 59-year-old man was admitted with pyrexia, chest pain, and dyspnea. A chest radiograph revealed a pneumonia shadow in the left lung field. Computed tomography (CT) revealed a lymph node-like shadow beside the aortic arch (Figure 1, A). He had progressive leukocytosis of $47.3 \times 10^{9} / \mathrm{L}$ (normal range, 3.5-9.5 $\times$ $109 / \mathrm{L}$ ), a C-reactive protein level of $28.6 \mathrm{mg} / \mathrm{dL}$ (normal, $<0.5$ $\mathrm{mg} / \mathrm{dL}$ ), and a hemoglobin A1c of $14.9 \%$. The results of blood cultures were positive for Streptococcus agalactiae. The chest radiograph revealed a gradual expansion of the aortic arch. CT and angiography revealed a huge thoracic aneurysm in the distal part of the aortic arch involving the left subclavian artery (Figure 1,B). He was finally operated on about 4 months after admission.

Both axillary arteries were exposed and anastomosed with 8-mm-diameter synthetic grafts for the outflow. After a median sternotomy, cardiopulmonary bypass was established, and right axillary arterial perfusion was used. During the hypothermic circulatory arrest at a body temperature of $20^{\circ} \mathrm{C}$ and antegrade cerebral perfusion with both axillary arterial grafts, which we routinely use to protect the brain, ${ }^{1}$ we transected the aortic arch between the brachiocephalic artery and the left common carotid artery. Left common carotid arterial perfusion was then begun selectively. A stent graft, which was constructed from a $30 \times$ 50-mm self-expandable Gianturco Z-shaped stent (William Cook Europe, Bjaeverskov, Denmark), with the distal part covered with a 24-mm collagen-coated woven Dacron graft, was delivered into the descending aorta by using exclusive delivery forceps, with the distal end located about $5 \mathrm{~cm}$ below the aneurysm, under monitoring with transesophageal echocardiography. The proximal end

From the Department of Cardiovascular Surgery, ${ }^{\text {a }}$ Urasoe General Hospital, Okinawa, Japan, and the Department of Surgery, Division of Cardiovascular Surgery, ${ }^{\mathrm{b}}$ Nippon Medical School, Tokyo, Japan.

Received for publication May 18, 2006; revisions received June 22, 2006; accepted for publication July 12, 2006.

Address for reprints: Hideyuki Iwaki, MD, Department of Cardiovascular Surgery, Urasoe General Hospital, 4-16-1 Iso, Urasoe, Okinawa, 901-2132 Japan (E-mail: iwaki@nms.ac.jp).

J Thorac Cardiovasc Surg 2006;132:1462-3

$0022-5223 / \$ 32.00$

Copyright () 2006 by The American Association for Thoracic Surgery doi:10.1016/j.jtcvs.2006.07.030 of the stent graft was sutured to the wall of the transected aortic arch. A 24-mm collagen-

coated woven Dacron graft with 4 branches was connected to the stent graft, and the proximal anastomosis was completed. During systemic perfusion, the heart was reperfused. During systemic and selective cerebral perfusion, 2 arch vessels, the brachiocephalic artery and the left common carotid artery, were reconstructed with the branches of the graft. Then the graft to the left subclavian artery was anastomosed to the third branch through the second intercostal space because the aortic aneurysm included the origin of the left subclavian artery. The cardiopulmonary bypass, aortic crossclamp, and circulatory arrest times were 272 minutes, 89 minutes, and 56 minutes, respectively. The postoperative course was essentially good. The patient was alive and well at 22 months after the operation, with no further evidence of infection. A CT scan in the early postoperative stage revealed that no endoleaks existed, and complete exclusion of the aneurysm was obtained
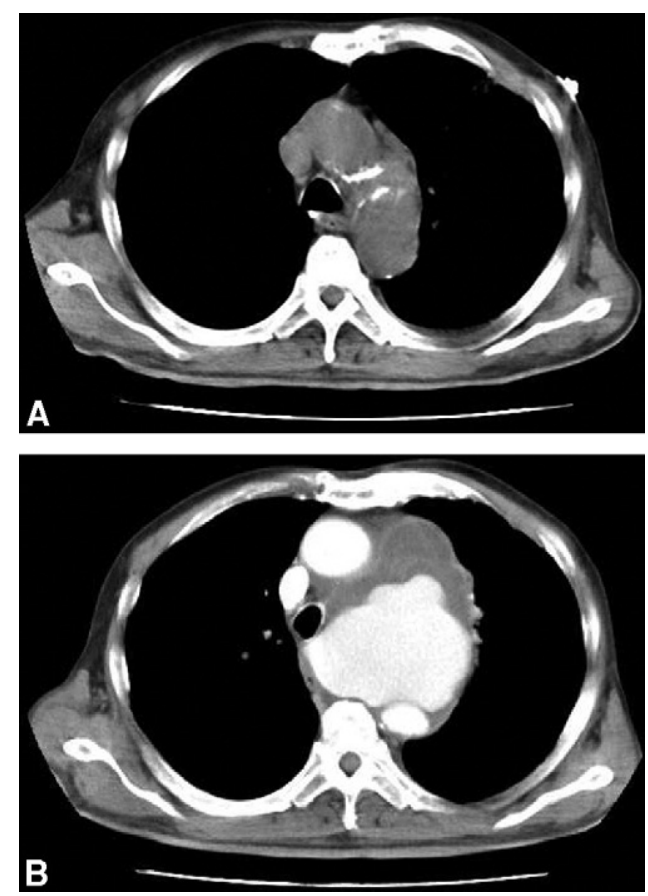

Figure 1. A, Chest computed tomographic scan on admission showing a lymph node-like shadow beside the aortic arch. B, Chest computed tomographic scan 2 months after admission showing a large aortic arch aneurysm. 

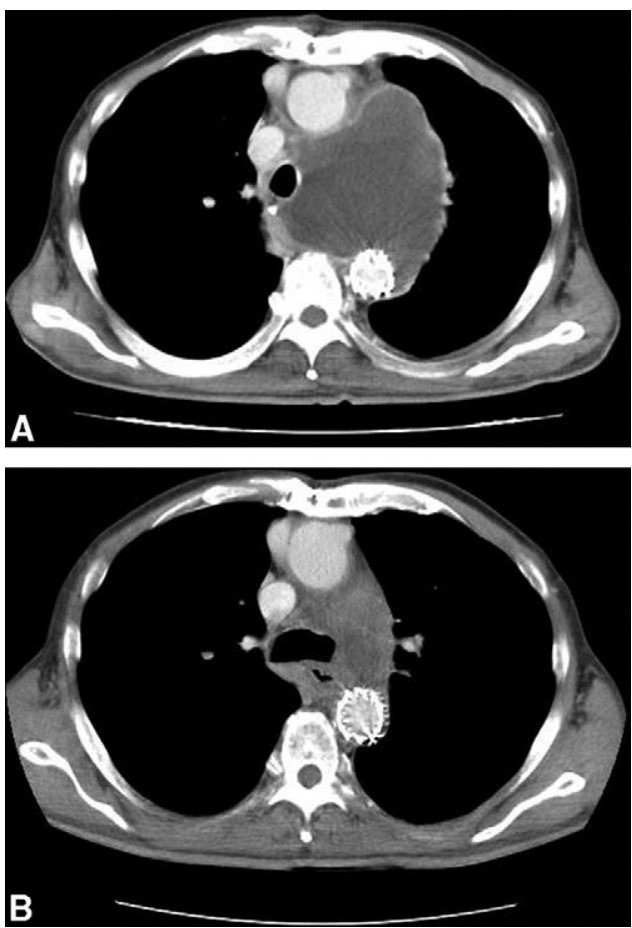

Figure 2. A, Chest computed tomographic scan during the early postoperative stage showing that there was no endoleak and complete exclusion of the aneurysm was obtained. B, Follow-up chest computed tomographic scan 7 months postoperatively showing that the aortic aneurysm had mostly disappeared.

(Figure 2, A). On a follow-up CT scan 7 months postoperatively, the aortic aneurysm had mostly disappeared (Figure 2, B).

\section{Discussion}

Open stent grafting used for aortic arch aneurysms, first reported by Kato and colleagues, ${ }^{2}$ has several advantages over a conventional synthetic replacement. Substitution of a stent graft placement for a distal anastomosis has reduced the operative risk, operative time, bleeding, and injury to adjacent organs. There are
2 main surgical strategies for mycotic aortic aneurysms: aortic exclusion and extra-anatomic bypass or surgical replacement of in situ grafts with a surgical resection of the infected tissue, including the aneurysm. However, there is no definite consensus on the surgical therapy. There have been no reports in which open stent grafting was used in mycotic aortic arch aneurysms. However, there are a few reports in which good results without surgical resection with endovascular stent grafting were obtained in spite of active infections. ${ }^{3,4}$ However, we abandoned the endovascular stent grafting in this patient because the aortic aneurysm included the origin of the left subclavian artery.

This patient showed no evidence of an active infection, and the results of blood cultures were negative without the use of any antibiotics before the operation, but he was not a desirable patient for a conventional surgical repair because he was very debilitated, as evidenced by a remarkable weight loss (body mass index, 19.5) and his being completely bedridden because of too long of a hospitalization. Furthermore, he had lost sight in his right eye during his hospitalization because of infectious uveitis. In addition, because his mycotic aneurysm became so large that it was pressing on many important mediastinal organs, such as the trachea, bilateral main bronchi, and esophagus, we realized that it was impossible to completely remove the infected tissue, and therefore we took the risk of selecting this procedure to achieve a less invasive operation.

Consequently, we suggest the use of open stent grafting for mycotic distal aortic arch aneurysms as an alternative treatment in patients who are high-risk candidates for a conventional surgical repair.

\section{References}

1. Usui A, Fujimoto K, Ishiguchi T, Yoshikawa M, Akita T, Ueda Y Cerebrospinal dysfunction after endovascular stent-grafting via a median sternotomy: the frozen elephant trunk procedure. Ann Thorac Surg. 2002;74(suppl):S1821-4.

2. Kato M, Ohnishi K, Kaneko M, Ueda T, Kishi D, Mizushita T, et al. New graft-implanting method for thoracic aortic aneurysm or dissection with a stented graft. Circulation. 1996;94(suppl):II188-93.

3. Semba CP, Sakai T, Slonim SM, Razavi MK, Kee ST, Jorgensen MJ, et al. Mycotic Aneurysms of the thoracic aorta: repair with use of endovascular stent-grafts. J Vasc Interv Radiol. 1998;9:33-40.

4. Ruth CVD, Jim R, Bas AJMDM, Hung O, Ron B. Aortoesophageal fistula secondary to mycotic aneurysm: endovascular repair and transhiatal esophagectomy. J Endovasc Ther. 2002;9:212-7. 